\title{
FlexAbility - Modeling and Maximizing the Bidirectional Flexibility Availability of Unidirectional Charging of Large Pools of Electric Vehicles
}

\author{
Jonas Schlund \\ jonas.schlund@fau.de \\ Friedrich-Alexander-University \\ Erlangen, Germany
}

\author{
Marco Pruckner \\ marco.pruckner@fau.de \\ Friedrich-Alexander-University \\ Erlangen, Germany
}

\author{
Reinhard German \\ reinhard.german@fau.de \\ Friedrich-Alexander-University \\ Erlangen, Germany
}

\begin{abstract}
We propose a new methodology for modeling flexibility availability (FlexAbility) of decentralized electric loads, e.g., electric vehicle charging, with an intuitive visualization method. The approach includes a novel method for aggregating and disaggregating flexibility that is more accurate and less complex than existing approaches. In addition, it is suitable for online flexibility determination and dispatch. It is the first which enables to consider a total energy constraint per individual load. We enable the determination of guaranteed aggregated FlexAbility over a time horizon by means of calculating flexibility dispatch paths. We then propose a method for maximizing the bidirectional power flexibility of unidirectional charging for generic applications in the power grid. We combine both new methods in a simulation model of electric vehicles with realistic mobility behavior. We are the first to provide an evaluation of the bidirectional power flexibility from unidirectional charging of electric vehicles, which is found to be bounded by the minimal capability to decrease charging power. We show that there is a trade-off between power and energy flexibility. Today, 20 thousand of the typical electric vehicles in Germany are able to keep bidirectional power flexibility of at least $1.3 \mathrm{MW}$ available during a whole year. The general modeling approach is applicable for other flexible loads with flexible profiles and a total energy constraint as well.
\end{abstract}

\section{CCS CONCEPTS}

- Hardware $\rightarrow$ Smart grid; • Computing methodologies $\rightarrow$ Modeling and simulation; Discrete-event simulation; $\bullet$ Mathematics of computing $\rightarrow$ Mathematical analysis.

\section{KEYWORDS}

electric vehicles, smart charging, flexibility, simulation

\section{ACM Reference Format:}

Jonas Schlund, Marco Pruckner, and Reinhard German. 2020. FlexAbility Modeling and Maximizing the Bidirectional Flexibility Availability of Unidirectional Charging of Large Pools of Electric Vehicles . In The Eleventh ACM International Conference on Future Energy Systems (e-Energy'20), Fune 22-26, 2020, Virtual Event, Australia. ACM, New York, NY, USA, 12 pages. https://doi.org/10.1145/3396851.3397697

This work is licensed under a Creative Commons Attribution International 4.0 License.

e-Energy'20, 7une 22-26, 2020, Virtual Event, Australia

(c) 2020 Copyright held by the owner/author(s).

ACM ISBN 978-1-4503-8009-6/20/06.

https://doi.org/10.1145/3396851.3397697

\section{INTRODUCTION}

The energy transition towards renewable energies is characterized by an increasing share of volatile, distributed energy resources (DERs). In order to continuously guarantee a high availability and stability of the electrical power system (EPS), different ancillary services for frequency stability, voltage stability or congestion management are necessary. As such flexibility services are mostly provided by conventional power plants, novel flexibility sources are necessary in order to realize the energy transition. As defined by [13], flexibility in an EPS is the ability to take different courses of action at a given point in time and thereby provide a service to third parties. Similarly, the authors of [5] describe flexibility as the set of all feasible load profiles for a given time frame.

According to the Global EV Outlook [11] electric mobility is expanding at a rapid pace. The global EV fleet exceeded 5.1 million in 2018 and China is the world's largest EV market with 2.3 million units. Also the number of EV chargers raised to an estimated 5.2 million in the same year. Forecasts in [11] show that the worldwide EV stock will increase to 250 million electric vehicles (EVs) in 2030 based on the EV30@30 Scenario.

While the integration of EVs in the EPS is a challenge itself [15], it is also an opportunity as an additional flexibility source. Assuming an average battery size of $30 \mathrm{kWh}$ of an EV, we obtain a total battery capacity of up to 7.5 TWh in 2030. Undoubtedly, a large proportion of that capacity can be used to make supply and demand more flexible and the provision of several system services for the EPS is possible [3,21]. In a future EPS with a high number of mobile battery storage systems we need to be able to quantify and optimally dispatch this flexibility.

For clearly defined goals with known data for both the mobility behavior and the flexibility need of the application in the EPS, an optimization of the charging power profiles of all participating vehicles is often sufficient. This way, e.g., peak shaving, renewable optimization, minimization of carbon emissions [24] or energy arbitrage [26] can be achieved without a dedicated flexibility model. If the application is not predictable a common approach in simulations is a flexibility provision without active or with simple rule-based flexibility management like in [22].

However, for the best use of the flexibility for ancillary services or multi-purposes it is necessary to actively manage the flexibility availability (FlexAbility) itself, independent from the application in the EPS. In addition, in order to be able to offer and monetize flexibility in markets the flexibility needs to be predictable, available and guaranteed over a defined period of time. In many cases the flexibility is required to be guaranteed in both directions (positive 
and negative flexibility). For the case of EVs the question arises how the charging flexibility can be modeled and how it can be dispatched in order to maximize its availability. The case of EVs is complex as it is characterized by uncertainty from the mobility behavior of EVs, i.e., when they arrive at electric vehicle supply equipments (EVSEs), which state of charge (SOC) they have upon arrival, how much energy they need to charge and when their next departure will be. The constraints from the mobility demand are more important than the charging flexibility as the primary use of an EV is mobility. In addition, EV charging is not only constrained by possible uncertain time limits but also by power limits and by energy constraints.

Bidirectional flexibility with unidirectional charging is interesting in particular as it has lower investment costs due to lower hardware requirements and does not add additional charging cycles to the battery, which would involve additional operational costs $[4,30]$. In contrast to bidirectional charging, flexibility from unidirectional charging has marginal costs close to zero if the EVSEs have communication and smart charging capabilities. However, unidirectional charging is also more constrained than bidirectional charging, which makes it even more important to optimally schedule and dispatch it.

This paper contributes to the state-of-the-art by proposing and evaluating an extended and enhanced FlexAbility model for the example of unidirectional EV charging based on related state-ofthe-art approaches. Our approach enables an accurate aggregation of flexibility of EV charging processes, aggregated processing and a disaggregation method into charging signals. In addition, we use this model as a basis for an adaptive control strategy that allows to determine a charging schedule ensuring the availability of power flexibility at all times in an environment with uncertainty.

Therefore, the paper is structured as follows. Sec. 2 summarizes related work considering large scale EV fleet charging dispatch and flexibility modeling. Sec. 3 describes our approach to model FlexAbility for the example of EV charging. Sec. 4 proposes power flexibility maximizing control strategies and evaluates the power flexibility of a large EV pool and Sec. 5 concludes the paper.

\section{RELATED WORK}

This section summarizes literature concerning optimization and dispatch strategies for large scale EV fleets before presenting related work considering flexibility modeling.

\subsection{Large scale EV fleet charging dispatch}

Several papers have investigated charging control of large populations of EVs, e.g., [14, 16, 23, 25]. The potential of decentralized charging control approaches for large scale EV fleets is explored by [14]. Real-time marginal electricity price information is utilized, which allows each EV to implement its own local charging control strategy aiming to minimize its individual costs of charging. It is shown that each EV reacts optimally with respect to a known charging trajectory, which is the average of all EV charging strategies under the existence of a unique Nash equilibrium. The authors give several numerical examples to demonstrate the performance of the proposed decentralized algorithm. One limitation of the algorithm is the absence of explicit time constraints, which makes such approaches infeasible for ancillary service provision with strict reliability and time constraints.

In [25] the authors present a centralized approach for EV fleet charging control by calculating individual charging schedules for each EV from an aggregator's perspective. The vehicle fleet consists of $2200 \mathrm{EVs}$ and each EV has its own driving behavior and vehicle parameters. In their paper the authors are mainly focused on the description of the associated optimization problems under consideration of electricity grid constraints. Hence, the resulting charging plans for each EV avoid distribution grid congestion while satisfying EV-owner service-level agreements. One limitation of the paper is the restricted consideration of randomness and prediction errors.

Another optimization-based approach is introduced by [23]. The authors propose a hierarchical charge control framework for large populations of EVs based on Benders decomposition. The upper level of the decomposition describes the minimization of the generation costs such that grid, units and EV aggregator constraints are fulfilled. EV constraints such as trip constraints or battery constraints are considered in the sub-problems on the lower level. The authors verify their framework by different case studies on an IEEE Reliability Test System.

For such optimization based approaches however, an optimization of large fleets with all the contraints of the individual vehicles require a lot of data and good predictions for all constraints and involved parameters. Moreover, the optimization itself can become computationally expensive. In addition, the application itself has to be known beforehand and it is not possible to optimize the FlexAbility. For ancillary services a spontaneous flexibility dispatch has to be possible at any given time. In order to realize this we built upon the ideas of [16]. They propose a least-laxity-first online algorithm. The authors show that it has a significantly higher rate of generating feasible EV charging decisions than several other common EV charging algorithms.

In addition to the least-laxity-first approach, we use a flexibility model to actively manage the FlexAbility itself without knowing the application beforehand. We propose a novel control method for large EV fleets suitable for online control and with a determination of a constant charging baseline that maximizes power flexibility. To our knowledge there is no other study or model which achieves such an active FlexAbility management for charging large fleets of EVs.

\subsection{Flexibility models for EV charging}

Concerning flexibility modeling a simple approach is to look at a fleet of EVs and aggregate them in the manner of a large storage tank. Such an approach of an equivalent time-variant storage (TVS) model is presented in [20]. Arrivals or departures of vehicles at charging stations of the virtual power plant (VPP) result in a change of the energy, capacity and the power limits of the TVS, which is implemented by means of impulse functions. The approach is suitable for an aggregated view of the momentary state of the VPP in terms of energy, power, capacity and power limits and also for forecasting. However, the model alone is not sufficient to evaluate the FlexAbility as the interdependency of both energy and power limits over time is missing. It offers no knowledge about how the 
state will change in case positive or negative flexibility is provided as it does not include the constraints of individual EVs. Concerning the disaggregation to control signals for individual EVs the authors propose an optimization. While this works for smaller fleets or in simulations, this approach is not scalable for a disaggregation of a large fleet when providing time sensitive ancillary services in online operation.

A general framework for demand side flexibility is presented in [1]. The framework defines a general notation suitable for many processes. However, it is not applicable for the detailed modeling and evaluation of EV charging flexibility as it does not take into account a fully flexible load with total energy constraints (upper and lower bounds) and charging power constraints. Moreover, it does not provide an aggregation method to enable aggregated processing.

A more detailed flexibility modeling framework is proposed in $[18,19,27-29]$. The authors characterize the flexibility of any process by means of objects called Flex-Offers (FOs). The framework implies discrete consumption or production profiles with minimum and maximum charging amounts for each time slice. In addition, they define the time flexibility as the difference between latest and earliest starting time of the profile. The energy flexibility is the sum over all slices of the difference between the maximum and minimum charging amount. They also propose complex methods for aggregation and disaggregation of FOs in [29] and methods which can handle interdependencies of the power limits in neighboring time slices [28]. More abstract, surrogate approaches using cascade classification and decoders to describe flexibility for VPP scheduling $[2,6,17]$ are not yet applicable in online applications.

The idea of FOs is generic and mature and is thus used as a basis for modeling flexibility in this paper. However, in order to quantify EV charging flexibility from many EVs and to control the charging processes of a large fleet of EVs under uncertain travel behavior in a manner that ensures available flexibility at all times there are still challenges to solve. In this paper, we simplify and extend the ideas and notations of the presented related work in order to be able to describe, aggregate and disaggregate the FlexAbility from EV-charging or other flexible loads characterized by total energy constraints in a scalable way. The contribution of this paper to the state-of-the-art of flexibility modeling is overcoming these four major challenges:

- Performance of the aggregation: The proposed aggregation methods are complex approximations, not accurate and lose too much flexibility or do not scale well [28]. Their simplified low-complexity model still only retains about $50 \%$ of the flexibility [28].

- Simplicity in formulation: The use of energy constraints for every time slice is generic and probably suitable for many applications. For flexibility sources like EV charging, the load can be adjusted within the operational limits with a fast response time. At least for slow charging the power limits are considered as constant during a charging event. There is neither a fixed profile nor an interdependency of neighboring time slices. However, there is a constraint concerning the total energy amount to be charged within a given time. Thus, total energy amount constraints are necessary and the constrains per slice become unnecessary overhead, which allows for a simplified notation for such loads.

- Aggregation method with individual total energy constraints: Although such additional total energy amount constraints for the formulation of optimizations are mentioned in $[19,27]$, we are not aware of any suitable aggregation and dissaggregation method that copes with such total energy amount constraints.

- Suitability for online control: The FOs are used for planning and scheduling of flexibility dispatch under the assumption that all constraints are known. A methodology which enables adaptive control in an environment with uncertainty is unknown to us. Therefore, the FlexAbility model uses timedependent values and a prioritized flexibility dispatch.

\section{FLEXABILITY MODEL}

For our understanding of flexibility in this paper we use, combine and extend approaches from Section 2.2. The TVS is used for the description of the momentary state and the FlexAbility, which builds upon the ideas of FOs, is used for the active flexibility management. This section describes the definitions of the FlexAbility for the example of individual charging processes, its aggregation methods and its disaggregation to control signals.

\subsection{FlexAbility of Individual Charging Processes}

We start by defining all basic variables before introducing a visualization method and the FlexAbility definition for individual charging processes. All basic definitions are also summarized in Tab. 1.

3.1.1 Basic Definitions. As the FlexAbility model is used in online operation in our simulations, the variables are time-dependent. Flexibility can only be available between the arrival time $t_{\text {arr }}$ of an EV at an EVSE and the departure time $t_{\mathrm{dep}}$. The total expected stay time at the EVSE $t_{\text {stay }}$ is defined in Eq. 1 . We define the remaining time duration until departure $\tilde{t}$ in Eq. 2 and the passed time duration since the arrival of the car at the EVSE as $t_{\text {past }}$ in Eq. 3. The remaining time duration until departure $\tilde{t}$ is a key variable and all following other variables that relate to that time duration are also marked with a superscript tilde.

$$
\begin{aligned}
t_{\text {stay }} & =t_{\text {dep }}-t_{\text {arr }} \\
\tilde{t}(t) & =t_{\text {dep }}-t \\
t_{\text {past }}(t) & =t-t_{\text {arr }}=t_{\text {stay }}-\tilde{t}(t)
\end{aligned}
$$

Concerning the energy amount to be charged during the whole charging process we define the minimum energy to be charged in order to satisfy the mobility demand as $\Delta E_{\min }$ and the maximum energy amount that is possible to charge due to the battery's capacity limit as $\Delta E_{\max }$. In Eq. 4 we define the already charged energy $\Delta E_{\text {past }}(t)$ at time $t . P(t)$ is the power which is actually charged. The minimum amount of energy that still needs to be charged during time $\tilde{t}$ in order to satisfy the following mobility demand is defined as $\Delta \tilde{E}_{\min }(t)$ and derived accordingly in Eq. 5. It is non-negative. In Eq. 6 the maximum amount of energy that can be charged during time $\tilde{t}$ is defined as $\Delta \tilde{E}_{\max }(t)$. It is also non-negative as $\Delta E_{\text {past }}(t)$ is 
upper bound by $\Delta E_{\max }$. In addition, $\Delta \tilde{E}_{\max }(t)$ is upper bound by the amount of energy that can be charged with charging power $P_{\text {max }}$ during time $\tilde{t}$. $P_{\max }$ is the maximum charging power of the EV at the EVSE and is asssumed to be constant. We reference $P_{\max } \cdot \tilde{t}$ as the total charging window size.

$$
\begin{aligned}
\Delta E_{\text {past }}(t) & =\int_{t_{\text {arr }}}^{t} P(x) d x \\
\Delta \tilde{E}_{\min }(t) & =\max \left(0, \Delta E_{\min }-\Delta E_{\text {past }}(t)\right) \\
\Delta \tilde{E}_{\max }(t) & =\min \left(P_{\max } \cdot \tilde{t}(t), \Delta E_{\max }-\Delta E_{\text {past }}(t)\right)
\end{aligned}
$$

Similar to the definitions from [18] we define the absolute energy flexibility $\Delta \tilde{E}_{\text {flex }}$ as the difference between the maximum and the minimum energy to be charged (Eq. 7). It can become negative if the duration $\tilde{t}$ is too short to charge $\Delta \tilde{E}_{\text {min }}$. In addition, we define the unused charging window size $\Delta \tilde{E}_{\text {free }}$ in Eq. 8 as the difference between the total charging window and the minimum energy to be charged.

$$
\begin{aligned}
& \Delta \tilde{E}_{\text {flex }}(t)=\Delta \tilde{E}_{\max }(t)-\Delta \tilde{E}_{\min }(t) \\
& \Delta \tilde{E}_{\text {free }}(t)=P_{\max } \cdot \tilde{t}(t)-\Delta \tilde{E}_{\min }(t)
\end{aligned}
$$

The minimum time needed to charge the minimal outstanding energy that is still missing is $t_{\min }$ as defined in Eq. 9. In [18] the authors define the time flexibility as the difference between latest start time $t_{\mathrm{ls}}$ and earliest start time $t_{\mathrm{es}}$. Similarly, the authors of [16] use the term laxity for the metric. We define the same metric in a time-dependent way as the absolute time flexibility $\phi_{\mathrm{abs}}$. A simple intuition for this metric is that, for any EV, there is a time when it must be charged at full power in order to be charged enough before it leaves. After this time, the time flexibility $\phi_{\text {abs }}$ becomes zero and the EV can show no flexibility. The earliest and the latest start time (for charging $\Delta \tilde{E}_{\min }(t)$ ) are time-dependent as they are influenced by the past charging decisions over time. As stated in Eq. 10, $\phi_{\text {abs }}$ is then equal to the difference between $\tilde{t}$ and $t_{\min }$.

$$
\begin{aligned}
t_{\min }(t) & =\frac{\Delta \tilde{E}_{\min }(t)}{P_{\max }} \\
\phi_{\mathrm{abs}}(t) & =t_{\mathrm{ls}}(t)-t_{\mathrm{es}}(t)=\tilde{t}(t)-t_{\min }(t)
\end{aligned}
$$

In addition to this absolute time flexibility, we define the relative time flexibility $\phi_{\text {rel }}$. It is defined in Eq. 11 as the ratio of the unused charging window size (Eq. 8) to the total charging window size. $\phi_{\text {rel }}$ is lower bound by zero and upper bound by one. All loads with $\phi_{\text {rel }} \geq 0.5$ are fully shiftable, i.e., the minimum energy to be charged fits at least twice in the charging window size.

$$
\begin{aligned}
\phi_{\text {rel }}(t) & =\frac{\Delta \tilde{E}_{\text {free }}(t)}{P_{\max } \cdot \tilde{t}(t)} \quad=1-\frac{\Delta \tilde{E}_{\text {min }}(t)}{P_{\max } \cdot \tilde{t}(t)} \\
& =1-\frac{t_{\min }(t)}{\tilde{t}(t)} \quad=\frac{\phi_{\text {abs }}(t)}{\tilde{t}(t)} \quad \text { with } \phi_{\text {rel }} \in[0,1]
\end{aligned}
$$

\begin{tabular}{|c|c|c|}
\hline Symbol & Description & Def. \\
\hline$t$ & model time & - \\
\hline$t_{\mathrm{arr}}$ & arrival time at the EVSE & - \\
\hline$t_{\mathrm{dep}}$ & departure time from the EVSE & - \\
\hline$t_{\text {stay }}$ & total stay time at the EVSE & Eq. 1 \\
\hline$\tilde{t}(t)$ & remaining time duration until departure & Eq. 2 \\
\hline$t_{\text {past }}(t)$ & passed time duration since arrival & Eq. 3 \\
\hline$t_{\min }(t)$ & min. time needed to charge $\Delta \tilde{E}_{\min }(t)$ & Eq. 9 \\
\hline$t_{\mathrm{es}}$ & earliest start time & - \\
\hline$t_{1 \mathrm{~s}}$ & latest start time & - \\
\hline$\Delta E_{\min }$ & min. energy to charge (mobility demand) & - \\
\hline$\Delta E_{\max }$ & max. energy to charge (capacity limit) & - \\
\hline$\Delta E_{\text {past }}(t)$ & already charged energy until $t$ & Eq. 4 \\
\hline$\Delta \tilde{E}_{\min }(t)$ & min. energy still to charge during $\tilde{t}(t)$ & Eq. 5 \\
\hline$\Delta \tilde{E}_{\max }(t)$ & max. energy still to charge during $\tilde{t}(t)$ & Eq. 6 \\
\hline$\Delta \tilde{E}_{\text {flex }}(t)$ & absolute energy flexibility & Eq. 7 \\
\hline$\Delta \tilde{E}_{\text {free }}(t)$ & unused charging window size & Eq. 8 \\
\hline$P_{\max } \cdot \tilde{t}$ & total charging window size & - \\
\hline$P(t)$ & actually charged power & - \\
\hline$P_{\max }$ & max. charging power of EV at EVSE & - \\
\hline$P^{+}(t)$ & $\begin{array}{l}\text { momentary positive power flexibility } \\
=\text { capability to decrease charging power }\end{array}$ & - \\
\hline$P^{-}(t)$ & $\begin{array}{l}\text { momentary negative power flexibility } \\
=\text { capability to increase charging power }\end{array}$ & - \\
\hline$\phi_{\mathrm{abs}}(t)$ & absolute time flexibility & Eq. 10 \\
\hline$\phi_{\mathrm{rel}}(t)$ & relative time flexibility & Eq. 11 \\
\hline$\psi_{\text {rel }}(t)$ & relative priority & Eq. 12 \\
\hline$\Phi(t)$ & FlexAbility of one charging process & Eq. 13 \\
\hline$\tau_{P}$ & flexibility threshold path for power $P$ & Eq. 14 \\
\hline$\tau_{P}\left(t_{i}\right)$ & threshold setting at $i$-th timestep on $\tau_{P}$ & Alg. 1 \\
\hline$t_{\max }\left(\tau_{P}\right)$ & maximum guaranteed time horizon of $\tau_{P}$ & - \\
\hline$T$ & aggregated FlexAbility model & Eq. 15 \\
\hline
\end{tabular}

Based on these time flexibility definitions we accordingly define the priority $\psi$ as a measure of the urgency of a load. The relative priority $\psi_{\text {rel }}$ as defined in Eq. 12 reflects the share of $\Delta \tilde{E}_{\min }(t)$ to the total charging window and is also upper bound by one and lower bound by zero. Accordingly, all loads with $\psi_{\text {rel }} \leq 0.5$ are fully
Table 1: Variable definition overview

shiftable.

$$
\psi_{\mathrm{rel}}(t)=1-\phi_{\mathrm{rel}}(t)=\frac{\Delta \tilde{E}_{\min }(t)}{P_{\max } \cdot \tilde{t}(t)}=\frac{t_{\min }(t)}{\tilde{t}(t)} \quad \text { with } \psi_{\mathrm{rel}} \in[0,1]
$$

3.1.2 FlexAbility Visualization with FlexBars. For a better intuition we define a visualization via FlexBars as shown in Fig. 1. Fig. 1 a) shows how the FlexBar is generally defined. The total width of the FlexBar is $\tilde{t}$. The minimum energy to charge $\Delta \tilde{E}_{\min }$ is the dark gray area on the left and can be charged with $P_{\max }$ in $t_{\min }$ (Eq. 9). The hatched area is equal to $\Delta \tilde{E}_{\text {flex }}$ (Eq. 7). The light gray area is the potentially unused or free charging window, which can be used for shifting. Its width equals $\phi_{\mathrm{abs}}$ (Eq. 10). Note that the FlexBar is time dependent and changes depending on the online charging decisions.

The relative time flexibility $\phi_{\text {rel }}$ is the share of the light gray (including hatched) area to the total area and the priority $\psi_{\text {rel }}$ is the share of the dark gray (excluding hatched) area to the total area. The total area equals the total charging window size. 


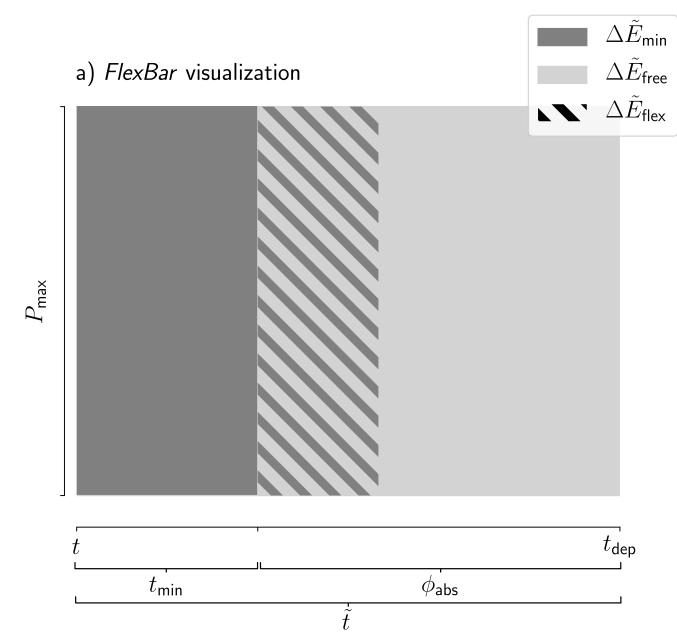

b) Low energy value (small $\left.\Delta \tilde{E}_{\max }\right)$

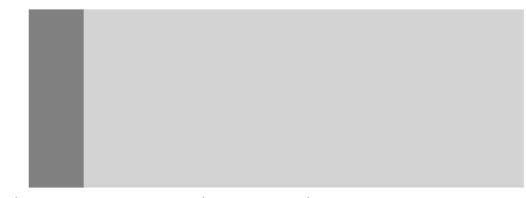

c) Low power value (small $\left.P_{\max }\right)$

d) Low time flexibility (small $\phi$ )

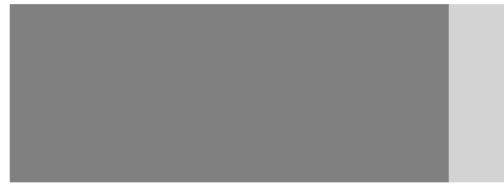

Figure 1: FlexAbility visualization with FlexBars: a) shows the definition and b) to d) show examples of low energy respectively power value and low time flexibility in a qualitative way.

Fig. $1 \mathrm{~b}$ ) to d) show different FlexBars with a low flexibility value in a qualitative way. While both absolute and relative time flexibility are high in b), its value for flexibility services is still low, as the shiftable energy amount is low. Accordingly, in c) the power is low, which also restricts its flexibility value. In d) both energy and power value are high. However, as the time flexibility is low, its usability for flexibility services is restricted.

3.1.3 FlexAbility Definition. As visualized in Fig. 1 b) to d), flexibility has to be observed in the three dimensions of power, energy and time. While $\phi$ quantifies the shiftability of a load (or $\psi$ the urgency), $\Delta \tilde{E}_{\max }$ and $P_{\max }$ quantify the value of the load. Thus, we define the total individual FlexAbility $\Phi$ (absolute or relative) in Eq. 13 with a tuple reflecting these three dimensions.

$$
\Phi_{\text {rel } / \text { abs }}(t)=<\phi_{\text {rel/abs }}(t), \Delta \tilde{E}_{\max }(t), P_{\max }>
$$

In comparison to the related work from Sec. 2, this definition enables a comparability of individual unidirectional loads in all the three important dimensions. A definition for bidirectional charging stations is possible accordingly, but out of scope here as this paper focuses on flexibility from unidirectional charging.

\subsection{FlexAbility Aggregation and Disaggregation}

In this section we use data from [12] of over 22 thousand real charging sessions for all visualizations used to explain the methodology. For this purpose we assume these charging sessions to happen concurrently and to have a maximum charging power of $7.4 \mathrm{~kW}$ per EVSE. For the FlexAbility aggregation we differentiate between the momentary flexibility availability and the flexibility availability over a time horizon.

For the momentary aggregated snapshot of many individual EV loads we use cumulative distribution functions (CDFs) of the available power respectively energy as shown exemplary in Fig. 2. All FlexAbilities are sorted based on their time flexibility and the cumulated available power respectively energy is calculated over

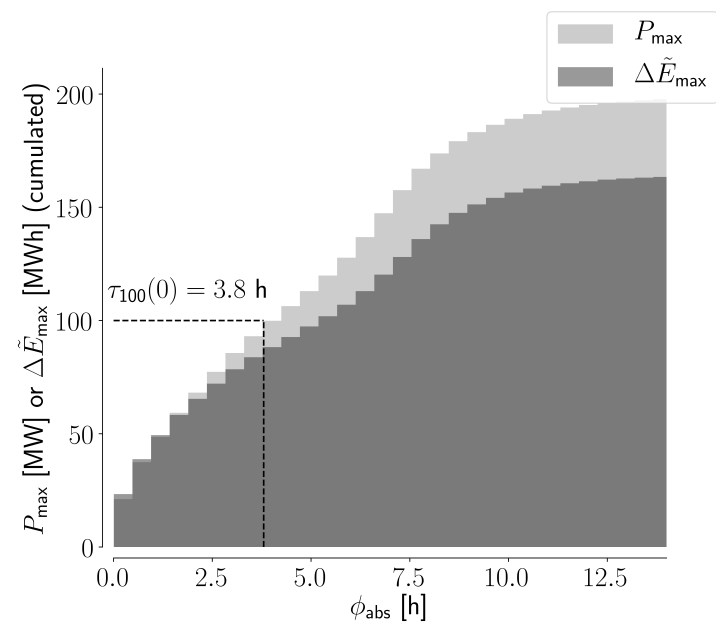

Figure 2: Momentary FlexAbility aggregation snapshot via cumulative distribution functions for $t_{1}=0$ with an exemplary determination of the flexibility threshold $\tau_{100}(0)$ of $3.8 \mathrm{~h}$ for a momentary power consumption of $100 \mathrm{MW}$.

the time flexibility. The idea behind this is to charge EVs with a higher urgency first, similar to [16]. The graphs in Fig. 2 show the CDF of the available power in light gray and the CDF of the available energy in dark gray. In case the aggregator wants its EV pool to consume a certain total power (e.g., $100 \mathrm{MW}$ ), he calculates the according time flexibility threshold (e.g., $3.8 \mathrm{~h}$ ). Depending on the preference either $\phi_{\text {rel }}$ or $\phi_{\text {abs }}$ can be used for this process. The control signal for each individual EVSE is calculated based on the threshold setting. This can be done centrally by the aggregator or even by the EVSEs themselves in a decentralized way. If an EV has a lower time flexibility than the threshold (e.g., $\phi_{\mathrm{abs}} \leq 3.8 \mathrm{~h}$ ), it charges at full power. Else it is not charging yet. For small EV 


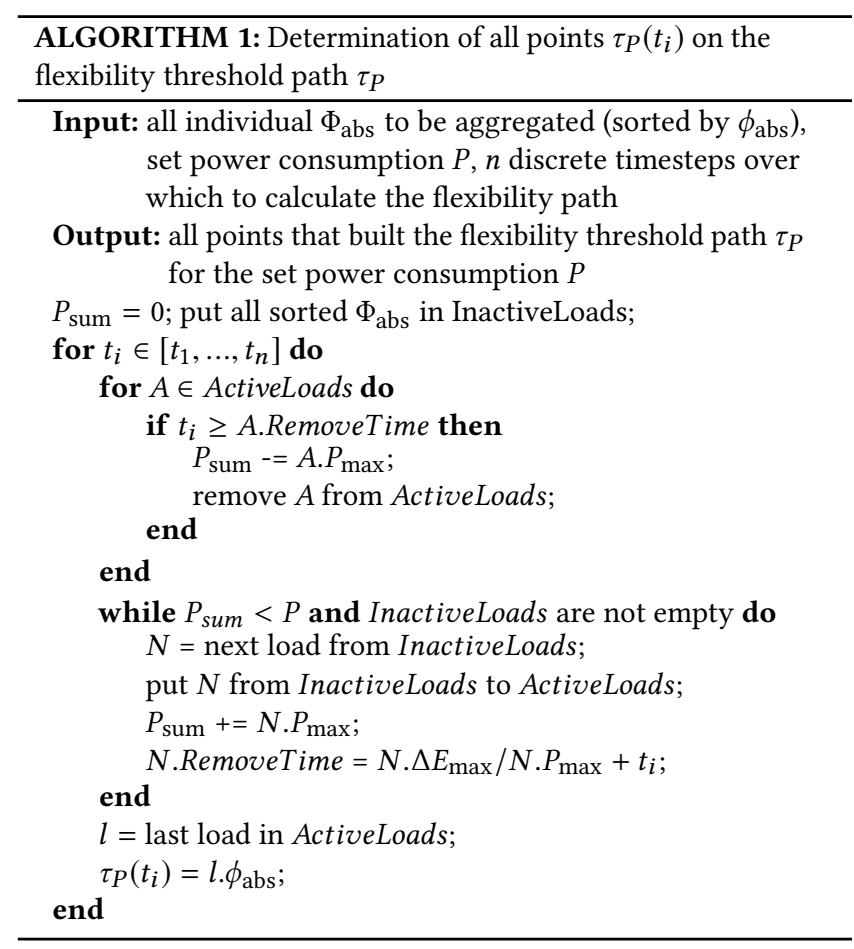

pools an additional correcting factor is necessary in order to enable an accurate cumulated power consumption. For large EV pools the resulting deviation is within measurement inaccuracy anyway. Note that, in contrary to offline optimization based approaches, this online methodology still works reasonably if the energy flexibility and thus also the time flexibility of some participating EVs becomes negative.

While this methodology is suitable a) to quantify the momentary availability of power and energy depending on $\phi$ and b) to derive control signals for all individual EVSEs in order to consume the correct cumulated amount of power, it does not quantify for how much time a chosen power consumption can be maintained. The information of the individual energy limits determine how long an individual load can maintain its power consumption and this information is lost if the aggregation is just done with the described CDFs. Thus, we calculate the resulting control path over a future time horizon in order to determine how long a power consumption can be maintained.

To determine for how much time a given power consumption $P$ is possible with a given set of EV charging events, we calculate flexibility threshold paths $\tau_{P}$ of the necessary flexibility threshold that will result in a constant consumption of the requested power $P$ over a time horizon. A flexibility threshold path starts at the current time $\left(t_{1}=0\right)$ at the flexibility threshold from the CDF of the flexibility aggregation snapshot in Fig. 2 and is calculated in discrete time steps according to Alg. 1 . Thus, $\tau_{P}\left(t_{i}\right)$ is the time flexibility threshold setting in order to provide $P$ at the $i$-th timestep of the time horizon. The flexibility threshold path $\tau_{P}$ is then the course of the setting of the threshold of $\phi_{\mathrm{abs}}$ in order to constantly provide $P$ over the time horizon and is formally defined as an array of the

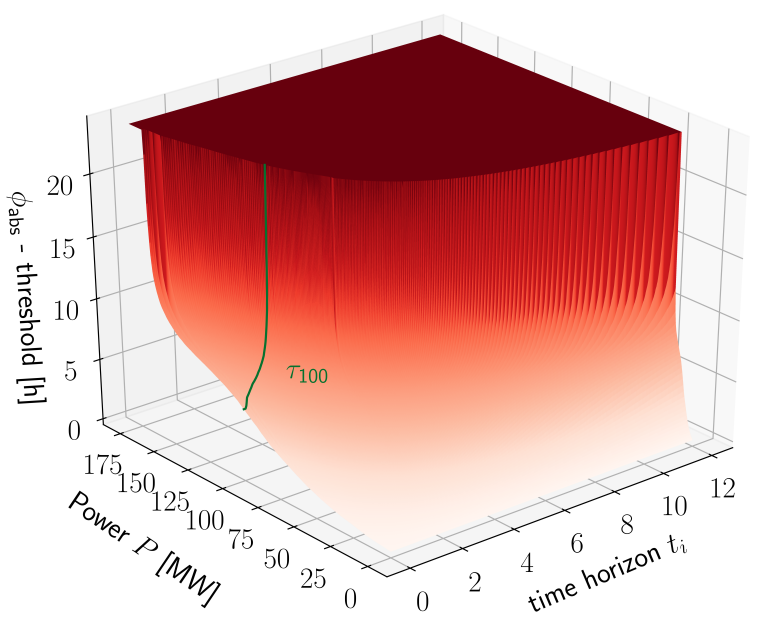

Figure 3: Exemplary visualization of the aggregated FlexAbility $T$ as a constant power flexibility paths area in the three dimensional time horizon, charging power and $\phi$-threshold space. An exemplary flexibility threshold path $\tau_{100}$ for the cumulated power consumption of $100 \mathrm{MW}$ is highlighted in green.

thresholds for each of the $n$ timesteps (Eq. 14).

$$
\tau_{P}=\left[\begin{array}{lll}
\tau_{P}\left(t_{1}\right) & \ldots & \tau_{P}\left(t_{n}\right)
\end{array}\right]
$$

Alg. 1 separates the loads in active and still inactive loads at the discrete time steps, i.e., loads that are already charging and loads that are not yet charging at a given timestep. At the beginning $\left(t_{1}=0\right)$ all the loads until reaching the time flexibility threshold from the CDF in Fig. 2 become active in order to consume the requested power. Once a load becomes active, the time when it will be fully charged (RemoveTime in Alg. 1) is determined based on the time when it is activated, its charging power and its energy that can be charged until it is full. At each timestep the fully charged EVs are removed. The missing power is then refilled by activating the next EV charging processes from the prioritized, still inactive loads. This way, the flexibility path $\tau_{P}$ is calculated as the necessary flexibility threshold to provide the requested power $P$ over the time horizon.

As defined in Eq. 15 the total FlexAbility aggregation model $T$ is a set of $m$ such flexibility threshold paths for different possible power consumptions. In the minimum case it just consists of one path. Starting from two paths, intermediate paths can be interpolated. A total picture of the available FlexAbility results from calculating many paths in a high enough resolution in both the power and the time horizon dimension as shown in Fig. 3. Additionally, one exemplary path for a consumption of the EV pool of $100 \mathrm{MW}$ is highlighted in green in Fig. 3. This path starts at a time flexibility threshold of $3.8 \mathrm{~h}$ as already determined in Fig. 2.

$$
T=\left\{\tau_{P_{1}}, \ldots, \tau_{P_{m}}\right\}
$$

Another constraint, which needs to be considered, is the lower power consumption limit. In case the requested power consumption $P$ is very low, it can occur that some EVs do not charge although 


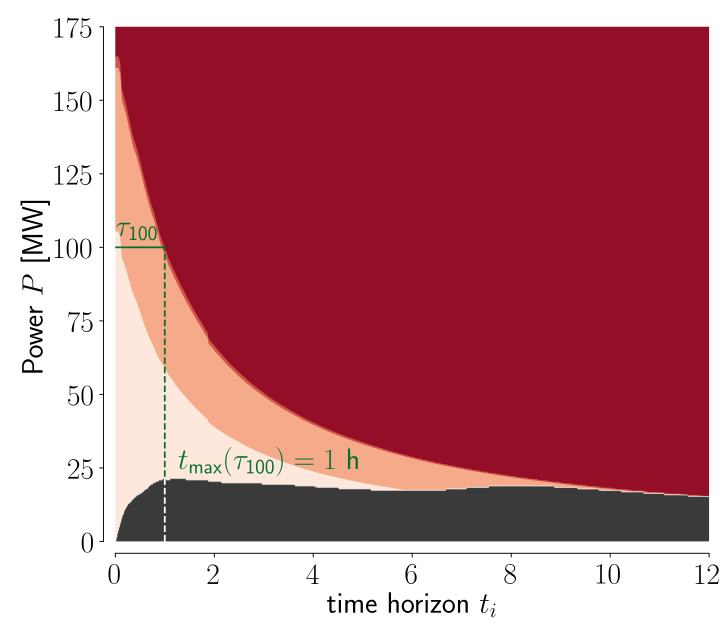

Figure 4: Contour of the exemplary visualization of the FlexAbility $T$ with a consideration of the upper (dark red) and lower (black) charging bounds. The maximum possible time horizon for $\tau_{100}$ (green line) is $1 \mathrm{~h}$ (dashed green line)

they need to charge to satisfy their mobility needs. These cases can be identified very simply if the absolute time flexibility is used for the prioritization. It happens whenever a path $\tau_{P}$ crosses a space where its value is smaller than the already passed time horizon $\left(\tau_{P}(t)<t\right)$. This means that the absolute time flexibility threshold until which loads are charged is smaller than the passed time, which results in some loads not being charged although they have no time flexibility left.

Fig. 4 shows a two dimensional projection of Fig. 3 in which the area with $\tau_{P}(t)<t$ is colored in black. The resulting contour plot visualizes the upper and lower power limits over time. The darkness of the red color indicates the threshold setting from low (light red) to high (dark red). The dark red area is not reachable as too many EVs are fully charged and thus the charging power can no longer be reached even with using all available EVs. On the other hand, the black area is not reachable as the charging power is not sufficient to satisfy the charging need of the mobility demand of all EVs. For each power value $P$ the maximum time it can be consumed constantly $t_{\max }\left(\tau_{P}\right)$ can be determined by means of the first intersection with either the dark red or the black area. Note that this is valid only if the set of connected EVs stays constant, so no new EVs arrive and EVs depart only as expected. Thus, the areas change with passing time and changes of the set of EVs. The maximum time for a consumption of $100 \mathrm{MW} t_{\max }\left(\tau_{100}\right)$ is determined in Fig. 4 as $1 \mathrm{~h}$.

Depending on the time horizon of a targeted flexibility service, guaranteed FlexAbilities of the unidirectionally charging EV pool can be derived based on Fig. 4. In this context, positive power flexibility $P^{+}$is the ability to spontaneously provide additional power to the grid, respectively reduce the planned power consumption of the EV pool. Accordingly, negative power flexibility $P^{-}$is the ability to reduce the power provision to the grid or to ramp up the load of the EV fleet. One example based on Fig. 4 might be the provision of only $P^{-}$over a time horizon of $3 \mathrm{~h}$. A well chosen baseline (has to be determined beforehand) for this service might be charging with $22 \mathrm{MW}$, as the lower bound is not going to be violated. Over $3 \mathrm{~h}$ a maximum constant charge of $50 \mathrm{MW}$ is possible, which leaves $28 \mathrm{MW}$ of guaranteed availability of $P^{-}$over $3 \mathrm{~h}$. In the case of biderectional flexibility provision $\left(P^{+}\right.$and $\left.P^{-}\right)$over $2 \mathrm{~h}$, the upper bound is $66 \mathrm{MW}$ and the lower bound is $22 \mathrm{MW}$. Thus, a good baseline is $44 \mathrm{MW}$, as it holds symmetrical power flexibility of $22 \mathrm{MW}$ available.

\section{MAXIMIZING POWER FLEXIBILITY OF EVs}

A problem not yet tackled in the sections before is how the baseline power for flexibility provision can be determined beforehand in a way which allows for a maximized bidirectional power flexibility. As shown in the example at the end of Sec. 3.2 it is possible to determine an optimal baseline whenever determining the aggregated FlexAbility $T$. However, in reality the baseline power for flexibility services often has to be determined beforehand, e.g., one day before. Thus, in this section we present novel strategies of determining a constant baseline power over a day, before evaluating the resulting momentary power flexibility over a short time horizon $\left(t_{1}=0\right)$ of the strategies over time by means of different simulations.

\subsection{Control Strategies}

In general we differentiate between fully decentralized strategies, where a local controller in the EVSE can make the charging decisions based on local information, and strategies with a central aggregator. In the second case the aggregator receives the necessary information of all the participating EVSEs in order to be able to use the aggregation and disaggregation methods proposed in Sec. 3.2. In addition, hybrid approaches are possible but out of scope here.

4.1.1 Fully Decentralized Strategies. The business-as-usual case for EV charging is uncoordinated charging (UC), where EVs start to charge at full power capability whenever they are plugged in at an EVSE and until they are fully charged. Modeling this behavior we differentiate between UC, where an EV is always plugged in when it arrives at an EVSE, and necessary charging (NC), where the EV is only plugged in if it actually needs to charge in order to satisfy its mobility demand until it will arrive at its main EVSE. NC assumes perfect foresight of the mobility behavior and consumption of the $\mathrm{EV}$. Both UC and NC do not offer any availability of $P^{-}$as it is never possible to ramp up the load from a UC or NC baseline. All EVs which are connected to an EVSE are either already charging at full power capacity or already fully charged.

An option to increase the availability of momentary $P^{-}$is to delay charging processes. In the case of delayed charging (DLC) an EVSE starts charging at maximum power at the latest possible time under the constraint that the needed energy for the mobility demand is still charged until the departure. Another option is levelized charging (LC), where the EV charges at full power from the time it arrives at the EVSE and until a certain SOC is reached. It then stops charging and switches to the DLC strategy. For example, 50-LC means immediate charging until a SOC of $50 \%$ before switching to DLC. Similar to with NC, a perfect foresight considering the constraints from the mobility behavior is assumed for LC and DLC.

As evaluated later in Sec. 4.2, these delaying strategies manage to free momentary $P^{-}$. However, they still lack a guaranteed, 
predictable baseline and they have a varying and sometimes very small $P^{+}$availability. Their cumulated charging profile varies heavily, which results in some timesteps with a very low maximum reducible power consumption.

4.1.2 Strategies with Central Coordination. For the conceptualization of the central strategies we assume the availability of data from the EVs at the EVSEs, e.g., via OCPP 2.0 and ISO 15118 or via the back-end of the car manufacturer. However, the according data of future arrivals is unknown to the aggregator. In addition, we require symmetrical power flexibility, which is bounded by the lower value of $P^{+}$and $P^{-}$. The following bottlenecks have to be solved in order to maximize the momentary power flexibility at any time. As shown in Fig. 7 and Fig. 8 and explained in Sec. 4.2, the bidirectional power flexibility from unidirectional charging is bounded by the non-availability of $P^{-}$in case of UC or NC and the minimum of the availability of $P^{+}$if charging processes are delayed. Thus, the symmetrical power flexibility for generic applications in the EPS is maximized by a) delaying charging processes in order to make $P^{-}$available and b) minimizing the variance of the charging power in order to maximize the minimum of $P^{+}$. In an ideal case this is achieved by a central strategy that charges at a constant rate over the considered period of time.

With a perfect foresight this problem can be solved by formulating an according optimization problem. As the data from EVs, which will arrive in the future, is unknown we propose an online adaptive controller to determine a constant baseline power for a specified time period $\Delta t$, e.g., a full day. The major constraint to be considered is that the total SOC of the whole pool of EVs stays within certain limits.

In Eq. 16-18 we define a digital PID controller with the nominal value of the target SOC $S O C_{n}$ as the command variable. The actual SOC $S O C_{a}$ of the pool of all EVs is the controlled variable. The task of the controller is it then to calculate the charging power baseline $P_{b}$ as the correcting variable. The controlled system is the behavior of the whole fleet with $P_{b}$ during $\Delta t$, with all kinds of disturbance variables like travel behavior, weather, holidays etc. The controller calculates $P_{b}$ according to Eq. 18 with:

- the timestep $t$ and previous timestep $t-1$

- the timestep size $\Delta t$ in [h]

- the total battery capacity of all connected EVs $C(t)$ e.g. in [MWh]

- the actual energy difference $\Delta E(t)$ at $t$ of the battery pool to the equivalent energy of $S O C_{n}$ with $C(t)$ in [MWh] as defined in Eq. 16

- an expected difference in energy consumption $\Delta E_{\exp }(t)$ in [MWh] between the timesteps (e.g. between fridays and saturdays)

- the ideal power difference $\Delta P_{b}(t)$ between the baseline power at timestep $t$ and $t-1$ as defined in Eq. 17

- a damping control gain $k \in[0,1]$ for setting the inertia of the controller
Table 2: Evaluated controller settings

\begin{tabular}{lcc}
\hline Parameter & Strategy A & Strategy B \\
\hline$\Delta t$ & $24 \mathrm{~h}$ & $24 \mathrm{~h}$ \\
$S O C_{n}$ & $60 \%$ & $60 \%$ \\
$k$ & 1.0 & 0.2 \\
$\Delta E_{\exp }$ & Sum of all $\Delta E(t)$ at the same day type & 0 \\
\hline
\end{tabular}

$$
\begin{aligned}
\Delta E(t) & =C(t) \cdot\left(S O C_{n}-S O C_{a}(t)\right) \\
\Delta P_{b}(t) & =\frac{\Delta E(t)-(\Delta E(t-1)-\Delta E(t))+\Delta E_{\exp }(t)}{\Delta t} \\
& =\frac{2 \cdot \Delta E(t)-\Delta E(t-1)+\Delta E_{\exp }(t)}{\Delta t} \\
P_{b}(t) & =P_{b}(t-1)+k \cdot \Delta P_{b}(t)
\end{aligned}
$$

The controller calculates the power baseline for the next $\Delta t$ by compensating the energy difference $\Delta E(t)$. The change of the energy difference $\Delta E(t-1)-\Delta E(t)$ during the last timestep is considered as well. This is the derivative term of the controller. In addition, an expected energy difference $\Delta E_{\exp }(t)$ can be considered, e.g., to charge with a smaller baseline on the weekends compared to the weekdays, as the consumption of the EVs is lower especially on sundays. The resulting ideal baseline power difference (Eq. 17) is then multiplied with the gain $k$ before it is added to the previously used $P_{b}$. The purpose of the gain $k$ is to control the inertia of the system, i.e., how fast the compensation towards $S O C_{n}$ is realized. While a large $k$ will compensate deviations faster it has also a higher tendency towards large deviations and possibly to overshooting due to the absence of damping.

As summarized in Tab. 2, we analyze the performance of two different controller settings. Both settings determine the baseline power over a full day. Strategy A fully focuses on days and aims at charging exactly the energy amount that is needed during a day. Thereby, the expected differences between the days are considered and $\Delta E_{\exp }(t)$ is set to the sum of all previously observed values of $\Delta E$ at the end of the same type of day. This is the integral term of the controller. Considered types of days are all seven days of the week and holidays. $\Delta E_{\text {exp }}$ is initialized based on previous simulations.

Strategy B additionally aims to compensate the varying charging consumption over the week. This is achieved by setting $\Delta E_{\text {exp }}$ to zero and decreasing the gain $k$ to 0.2 . This way, the baseline power adjustment is damped stronger and the variance of the baseline powers of different days is decreased. The total pool of EVs buffers deviations in consumption, which leads to a SOC above $S O C_{n}$ during times of lower consumption (e.g. on the weekends) but an overall less varying $P_{b}$. In the following section both settings are compared to UC and 50-LC and evaluated considering their momentary power flexibility over time.

\subsection{Evaluation}

For the evaluation we use discrete-event simulation with randomness with a timestep of 1 minute. We simulate 20 thousand typical EVs in Germany over a whole simulated year. We use an object oriented approach, where each EV is modeled as an individual instance of an abstract EV class. Each EV is assumed to have a main 


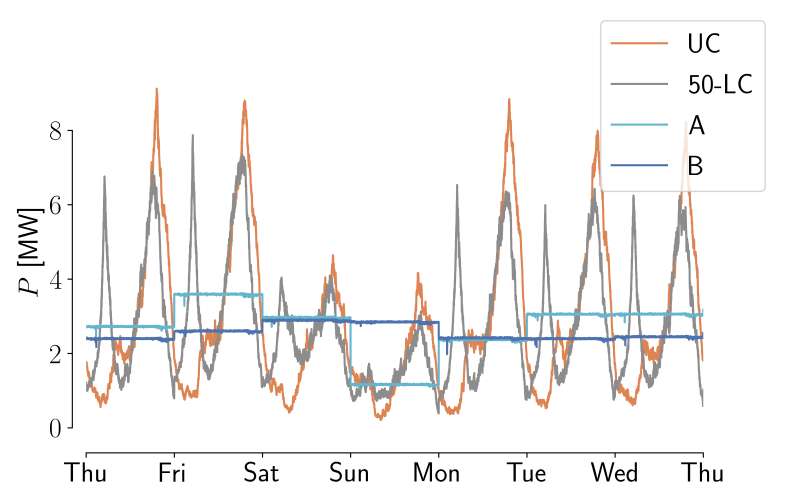

Figure 5: Courses of the cumulated charging power of 20 thousand EVs over an exemplary week in August. Orange is UC, gray is 50-LC and the blue curves are the two central control strategies.

EVSE, where it is primarily charged. Smart charging monitoring and control is only implemented at these main EVSEs. The main EVSEs are parameterized to be at homes for $75 \%$ and at workplaces for $25 \%$ of the EVs based on current estimates for the European Union [8]. Each main EVSE is assumed to have at least the same charging capacity as its corresponding EV. The EVs are parameterized according to the vehicle registration figures for EVs in Germany from Kraftfahrt-Bundesamt (Federal Motor Transport Authority) ${ }^{1}$, with technical parameters such as charging power limits, average consumption and battery capacities based on data from the car manufacturers. Based on this parameterization, the average battery capacity is $37.7 \mathrm{kWh}$ and the average charging power limit is $11.1 \mathrm{~kW}$. The mobility behavior of the EVs is modeled with purposebased trip chains of a stochastic length by means of a probabilistic representation of the mobility behavior of cars in Germany, based on the dataset B.1 of the Mobility in Germany report [10]. We use a Bayesian network with logical constraints to represent this dataset. The energy consumption of the EVs derives from the distance driven and is modeled in dependency of the ambient temperature and the driving speed based on data from [7]. It also includes the energy consumption of air conditioning. The model uses temperature data from the year 2013 in a 10 minute resolution from the Climate Data Center of Deutscher Wetterdienst (German Meteorological Service) ${ }^{2}$. The data is available for 87 weather stations in Germany and the ambient temperature of each EV is determined at each timestep based on the data from the closest weather station. In addition to charging at the main EVSE, secondary public EVSEs not participating in smart charging are available. Only when an EV leaves for a trip with a larger distance than its range with full battery capacity, the possibility of interim charging at such a secondary public EVSE is assumed. In reality an EV user would not start such a trip without the possibility for interim charging.

Fig. 5 shows the courses of the cumulated charging power of 20 thousand EVs at the monitored EVSEs for different charging strategies during an exemplary week in August. The orange line

\footnotetext{
$\overline{1_{\text {www.kba.de/EN/ }}}$

${ }^{2}$ https://cdc.dwd.de/portal/
}

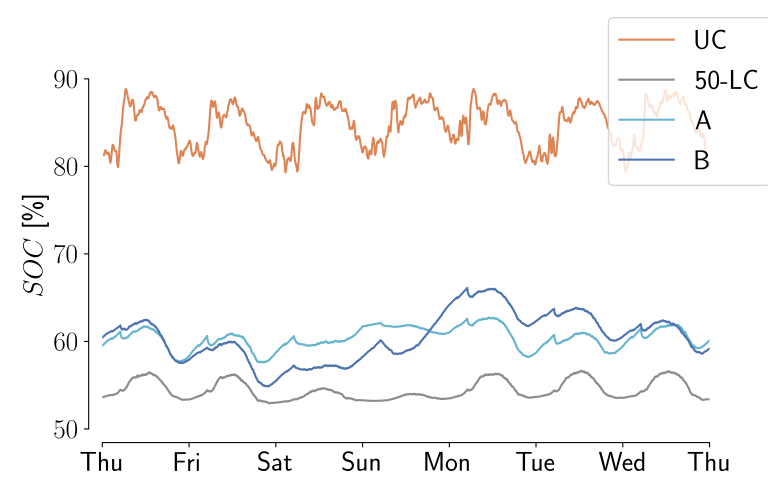

Figure 6: Courses of the SOC of the connected EVs of the pool over an exemplary week in August. Orange is UC, gray is 50-LC and the blue curves are the two central control strategies.

is UC and has peaks of more than $9 \mathrm{MW}$. These peaks occur in the late afternoon, when many EVs come back home from work. There is also a second small peak in the morning hours resulting from the proportion of EVs that charge at work. The profile shows that the demand is lower in general on the weekend. The gray curve shows the effect of 50-LC. As the EVs are charged to $50 \%$ SOC when they arrive and charged more just before departure in case they need it, the charging of an EV is split to two time periods. This results in slightly flatter peaks and valleys.

Fig. 6 compares the course of the SOC of the EV pool for the four control strategies during the same week. In case of UC the SOC course is always quite close to full batteries, while the course for $50-\mathrm{LC}$ is always a bit above the set level of $50 \%$. This is because there are always some EVs that need more charge and are thus charged to a higher SOC, which lifts the SOC of the pool a little bit. Note that the profiles are a little bit peaky as the total SOC of the pool is determined based on the energy to capacity ratio of the EVs currently connected to their main EVSEs.

As depicted in Fig. 5, it is possible to charge the whole fleet at a constant rate during a full day with both central charging strategies despite the constraints from the stochastic mobility behavior. As expected due to the controller settings from Tab. 2, strategy A has a differing charging power between the days and charges less on the weekends. Strategy B's charging power varies less between the days due its damped configuration. Looking at Fig. 6 we see that strategy B achieves this by letting the SOC drift between about 50 and $70 \%$. The controller of strategy A on the other hand acts more direct and lets the pool's SOC only differ between about 55 and $65 \%$. Summarizing, the strategy B buffers the differences in demand between the days using the storage capacity of the pool.

In order to evaluate the power flexibility over time, Fig. 7 and Fig. 8 provide insight. They show boxplots of the momentary negative respectively positive power flexibility of each minute during the whole month of August. An evaluation for a whole year is possible as well but needs a temporal resolution due to seasonal differences in consumption, which influence the average charging power. As this paper's main focus is on the methodology, an in-depth seasonal 


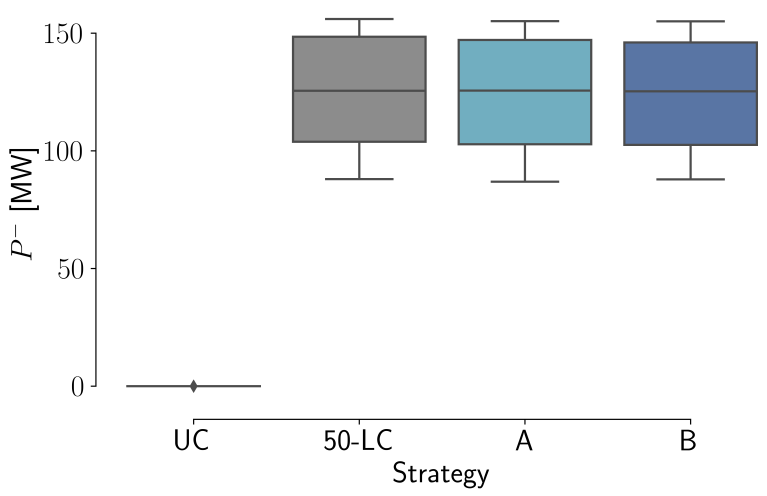

Figure 7: Boxplot of the momentary negative power flexibility $P^{-}$of 20 thousand EVs for each minute in August

analysis is out of scope here. Note that what looks like thick bars outside of the whiskers in the box plots are the outliers. The length of the whiskers is determined according to the default value of matplotlib [9] with 1.5 times the interquartile range.

As already mentioned in Sec. 4, Fig. 7 shows that UC never has any availability of $P^{-}$. All three other strategies free $P^{-}$mostly between 100 and $150 \mathrm{MW}$ as they manage to delay charging processes enough. Thus, 50-LC, which does not require any central coordination, is sufficient for an application that only requires $P^{-}$. Considering $P^{+}$in Fig. 8, it is not possible to shift the whole distribution upwards as its mean value is determined by the charging need, which is of course identical for all strategies. In the strategies with delayed charging, the bidirectional momentary power flexibility over the time is upper bound by the minimum of $P^{+}$as $P^{+}$is on a lower level than $P^{-}$. This minimum availability of $P^{+}$is maximized by minimizing the variance of the charging power.

While the distributions of UC and 50-LC have long tails in Fig. 8, the tails of the centralized strategies are shorter. The minimal momentary $P^{+}$is increased considerably by the central strategies. Strategy A has a higher variance than B as it has some days (e.g., the weekends) with less $P^{+}$. Strategy B manages to buffer differences between the days but also faces differences between the weeks due to its slow reaction of the damped control. Considering momentary power flexibility strategy B performs best with a minimum $P^{+}$of $1.7 \mathrm{MW}$. While $P^{-}$is not influenced considerably by the seasons, the $P^{+}$potential is in general higher in winter due to a higher consumption. Thus, evaluating $P^{+}$during the whole year leads to a higher and wider spread distribution with a lower quartile of 2.5 MW, a median of 3.1 MW and an upper quartile of 3.7 MW for strategy B. The minimal $P^{+}$for strategy B during the whole year was $1.3 \mathrm{MW}$ in our simulation, which is more than eight times the minimal $P^{+}$for UC.

However, the decreased variance in $P^{+}$from strategy B in comparison to strategy $A$ is only possible by means of buffering, using the storage capacity of the pool of EVs as seen in Fig. 6. Fig. 9 shows the boxplot of the SOC of the EV pool during the whole year. The central strategies both manage to keep the total SOC out of critical regions. The variance of strategy $B$ is higher than the variance of

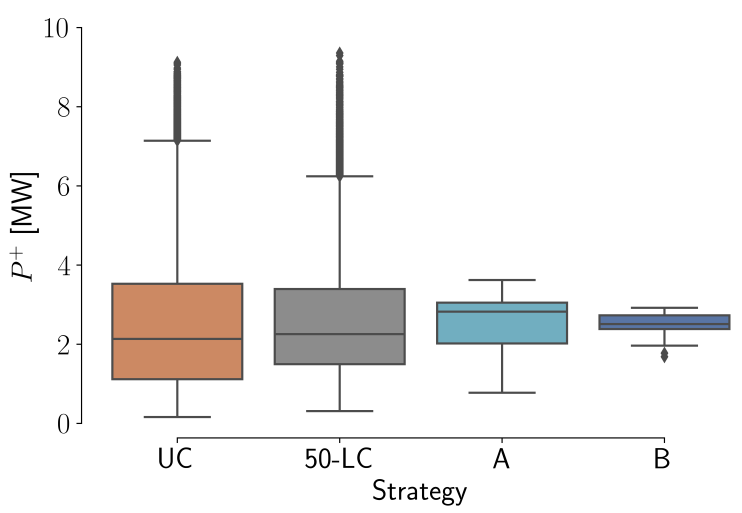

Figure 8: Boxplot of the momentary positive power flexibility $P^{+}$of 20 thousand EVs for each minute in August

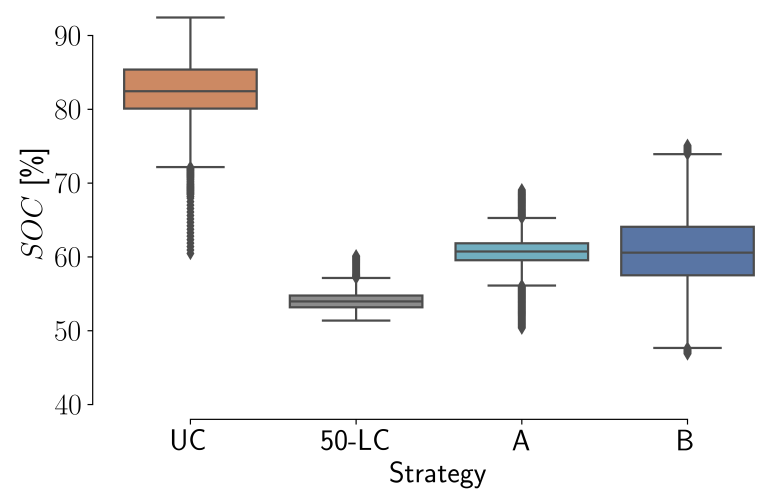

Figure 9: Boxplot of the SOCs of the EV pool for each minute during the whole simulated year

strategy A. This means that the minimal positive and negative energy flexibility is lower for strategy B compared to strategy A. Thus, there is a trade-off between available power flexibility and available energy flexibility. While a strategy focusing on a constant charging power maximizes the minimal momentary power flexibility, it does not achieve the same for the energy flexibility.

\section{CONCLUSION}

In this paper we propose and formally define the new concept of FlexAbility as the availability of flexibility in the dimensions of time, energy and power. We propose FlexBars for the visualization of the FlexAbility of individual loads and a novel method for aggregating loads and disaggregating the aggregated model to control signals. We define flexibility threshold paths to describe and control aggregated FlexAbility and to determine guaranteed FlexAbility over time of a given set of loads. We apply and evaluate the concept for EV charging. Furthermore, we propose and evaluate novel control strategies to maximize the momentary power flexibility of a pool of EVs under uncertainty from mobility behavior. The bidirectional power flexibility from unidirectional charging is bound by the minimal load reduction potential in case of all smart charging 
strategies. This is bound by the average consumption of the EVs, which leads to a higher flexibility potential in winter and will lead to a higher potential in future with larger and heavier EVs. For a fleet of 20 thousand typical EVs in Germany our simulations of the best control strategy show a minimal momentary power reduction potential during a whole year of $1.3 \mathrm{MW}$, which is eight times more than with uncoordinated charging. The potential to ramp up the load is almost two magnitudes higher. In general, there is a tradeoff between power and energy flexibility, which means that the baseline strategy of an aggregator has to be chosen based on the time horizon of the targeted flexibility services.

Summarizing, this paper's contribution are a) the methods of Sec. 3 which can be used to determine exactly how much flexibility availability can be guaranteed over any time horizon and b) the first extensive analysis of bidirectional flexibility from unidirectional charging from Sec. 4.2. Our proposed methods are evaluated for EV charging but also applicable for other loads characterized by minimum and maximum energy amounts that need to be consumed within a given time.

A limitation of the methodology is that a knowledge or an estimate of departure times and energy demands of the charging EVs is necessary to quantify the available momentary flexibility. Future work can extend current data-driven approaches [12] to predict these values and include an extensive evaluation of the performance of the methodology based on such estimations. In addition, the evaluation part is extensible in many directions. For a reliable quantitative evaluation for future scenarios we plan to include sensitivity analyses considering the technical vehicle data, the driving distances, the utilization of EVs, and temperatures. Part of our future work is a more detailed FlexAbility evaluation, as we so far only evaluated momentary power flexibility over time. In follow-up studies focusing on quantitative results we will evaluate FlexAbility over time (also considering the time horizon constraint) based on the methodology presented here. Furthermore, the prediction of the influence of probably arriving EVs on the FlexAbility model and dispatch under uncertain time flexibility are in our scope of further work.

Additionally, application focused studies with predictive flexibility dispatch for applications like redispatch, feed-in management or reserve power provision are interesting to evaluate considering their general potential for ancillary services and their cost or carbon emission savings. For such studies a review with seasonality, EV user groups and a simulation in geo-resolution is necessary, which is possible in our simulation model up to county level. As the proposed FlexAbility model of this paper does not include link capacities between parts of the EPS, our approach for such simulations is to use instances of the model for subgroups of the EVSE network. This way, an aggregator can individually control the flexibilities of each grid area and thus also consider link capacities by means of a superimposed model. Results of such studies can serve as a prerequisite for the design of incentives for participating EVs.

\section{ACKNOWLEDGMENTS}

This work is funded by the Bavarian State Ministry of Science and the Arts in the framework of the Centre Digitisation.Bavaria (ZD.B). We thank the reviewers for their careful reading and valuable input.

\section{REFERENCES}

[1] Lukas Barth, Nicole Ludwig, Esther Mengelkamp, and Philipp Staudt. 2018. A comprehensive modelling framework for demand side flexibility in smart grids. Computer Science - Research and Development 33, 1 (01 Feb 2018), 13-23. https: //doi.org/10.1007/s00450-017-0343-x

[2] Joerg Bremer and Sebastian Lehnhoff. 2018. A Cascading Chi-shapes based Decoder for Constraint-handling in Distributed Energy Management. In Proceedings of the 10th International Joint Conference on Computational Intelligence - Volume 1: IFCCI,. INSTICC, SciTePress, 184-191. https://doi.org/10.5220/0006926101840191

[3] Chris Develder, Nasrin Sadeghianpourhamami, Matthias Strobbe, and Nazir Refa. 2016. Quantifying flexibility in EV charging as DR potential: Analysis of two real-world data sets. In 2016 IEEE International Conference on Smart Grid Communications (SmartGridComm). 600-605. https://doi.org/10.1109/SmartGridComm. 2016.7778827

[4] Hossein Farzin, Mahmud Fotuhi-Firuzabad, and Moein Moeini-Aghtaie. 2016. A Practical Scheme to Involve Degradation Cost of Lithium-Ion Batteries in Vehicle-to-Grid Applications. IEEE Transactions on Sustainable Energy 7, 4 (Oct 2016), 1730-1738. https://doi.org/10.1109/TSTE.2016.2558500

[5] Kevin Förderer, Mischa Ahrens, Kaibin Bao, Ingo Mauser, and Hartmut Schmeck. 2018. Modeling flexibility using artificial neural networks. Energy Informatics 1 , 1 (2018), 21. https://doi.org/10.1186/s42162-018-0024-4

[6] Judith Fröhling. 2017. Abstract flexibility description for virtual power plant scheduling. Ph.D. Dissertation. Universität Oldenburg.

[7] Bernhard Geringer and Werner Tober. 2012. Batterieelektrische Fahrzeuge in der Praxis-Kosten, Reichweite, Umwelt, Komfort. Publikation des Österreichischen Vereins für Kraftfahrzeugtechnik (2012).

[8] Stefan Knupfer Shivika Sahdev Hauke Engel, Russell Hensley. 2018. Charging ahead: Electric Vehicle Infrastructure Demand. McKinsey Center for Future Mobility (2018)

[9] John D Hunter. 2007. Matplotlib: A 2D graphics environment. Computing in science \& engineering 9, 3 (2007), 90-95.

[10] infas, DLR, IVT and infas 360. 2018. Mobility in Germany.

[11] International Energy Agency. 2019. Global EV Outlook 2019 - Scaling-up the transition to electric mobility.

[12] Zachary J. Lee, Tongxin Li, and Steven H. Low. 2019. ACN-Data: Analysis and Applications of an Open EV Charging Dataset. In Proceedings of the Tenth International Conference on Future Energy Systems (e-Energy '19).

[13] Nico Lehmann, Emil Kraft, Clemens Duepmeier, Ingo Mauser, Kevin Förderer, and Dominique Sauer. 2019. Definition von Flexibilität in einem zellulär geprägten Energiesystem. In Zukünftige Stromnetze 2019: 30./31. Januar 2019 in Berlin. Conexio, Pforzheim, 459-469. 37.06.01; LK 01.

[14] Zhongjing Ma, Duncan. S. Callaway, and Ian. A. Hiskens. 2013. Decentralized Charging Control of Large Populations of Plug-in Electric Vehicles. IEEE Transactions on Control Systems Technology 21, 1 (Jan 2013), 67-78. https: //doi.org/10.1109/TCST.2011.2174059

[15] Yunfei Mu, Jianzhong Wu, Nick Jenkins, Hongjie Jia, and Chengshan Wang. 2014. A Spatial Temporal model for grid impact analysis of plug-in electric vehicles. Applied Energy 114 (2014), 456 - 465. https://doi.org/10.1016/j.apenergy.2013.10. 006

[16] Yorie Nakahira, Niangjun Chen, Lijun Chen, and Steven H. Low. 2017. Smoothed Least-Laxity-First Algorithm for EV Charging. In Proceedings of the Eighth International Conference on Future Energy Systems (e-Energy âĂŹ17). Association for Computing Machinery, New York, NY, USA, 242âĂȘ251. https: //doi.org/10.1145/3077839.3077864

[17] J. Neugebauer, J. Bremer, C. Hinrichs, O. Kramer, and M. Sonnenschein. 2016. Generalized cascade classification model with customized transformation based ensembles. In 2016 International foint Conference on Neural Networks (IFCNN). 4056-4063.

[18] Bijay Neupane, Laurynas Šikšnys, and Torben Bach Pedersen. 2017. Generation and Evaluation of Flex-Offers from Flexible Electrical Devices. In Proceedings of the Eighth International Conference on Future Energy Systems (e-Energy '17). ACM, New York, NY, USA, 143-156. https://doi.org/10.1145/3077839.3077850

[19] Torben. B. Pedersen, Laurynas Šikšnys, and Bijay Neupane. 2018. Modeling and Managing Energy Flexibility Using FlexOffers. In 2018 IEEE International Conference on Communications, Control, and Computing Technologies for Smart Grids (SmartGridComm). 1-7. https://doi.org/10.1109/SmartGridComm.2018. 8587605

[20] Michael Pertl, Francesco Carducci, Michaelangelo Tabone, Mattia Marinelli, Sila Kiliccote, and Emre C. Kara. 2019. An Equivalent Time-Variant Storage Model to Harness EV Flexibility: Forecast and Aggregation. IEEE Transactions on Industrial Informatics 15, 4 (April 2019), 1899-1910. https://doi.org/10.1109/TII.2018. 2865433

[21] Nasrin Sadeghianpourhamami, Nazir Refa, Matthias Strobbe, and Chris Develder. 2018. Quantitive analysis of electric vehicle flexibility : a data-driven approach. International fournal of Electrical Power \& Energy Systems 95 (2018), 451-462. http://dx.doi.org/10.1016/j.ijepes.2017.09.007 
[22] Jonas Schlund, Ronny Steinert, and Marco Pruckner. 2018. Coordinating EMobility Charging for Frequency Containment Reserve Power Provision. In Proceedings of the Ninth International Conference on Future Energy Systems ( $e$ Energy '18). ACM, New York, NY, USA, 556-563. https://doi.org/10.1145/3208903. 3213892

[23] Chengcheng Shao, Xifan Wang, Xiuli Wang, Chao Du, and Biyang Wang. 2016. Hierarchical Charge Control of Large Populations of EVs. IEEE Transactions on Smart Grid 7, 2 (March 2016), 1147-1155. https://doi.org/10.1109/TSG.2015. 2396952

[24] Martin Spitzer, Jonas Schlund, Elpiniki Apostolaki-Iosifidou, and Marco Pruckner. 2019. Optimized Integration of Electric Vehicles in Low Voltage Distribution Grids. Energies 12, 21 (2019). https://doi.org/10.3390/en12214059

[25] Olle Sundstrom and Carl Binding. 2012. Flexible Charging Optimization for Electric Vehicles Considering Distribution Grid Constraints. IEEE Transactions on Smart Grid 3, 1 (March 2012), 26-37. https://doi.org/10.1109/TSG.2011.2168431

[26] Hanif Tayarani, Hamidreza Jahangir, Razieh Nadafianshahamabadi, Masoud Aliakbar Golkar, Ali Ahmadian, and Ali Elkamel. 2019. Optimal Charging of Plug-In Electric Vehicle: Considering Travel Behavior Uncertainties and Battery
Degradation. Applied Sciences 9, 16 (2019). https://doi.org/10.3390/app9163420

[27] Emmanouil Valsomatzis, Katja Hose, Torben Bach Pedersen, and Laurynas Siksnys. 2015. Measuring and Comparing Energy Flexibilities. In Proceedings of the Workshops of the EDBT/ICDT 2015 foint Conference (EDBT/ICDT) (CEUR Workshop Proceedings), Peter M. Fischer, Gustavo Alonso, Marcelo Arenas, and Floris Geerts (Eds.), Vol. 1330. CEUR Workshop Proceedings, 78-85.

[28] Laurynas Šikšnys and Torben Bach Pedersen. 2016. Dependency-based FlexOffers: Scalable Management of Flexible Loads with Dependencies. In Proceedings of the Seventh International Conference on Future Energy Systems (e-Energy '16). ACM, New York, NY, USA, Article 11, 13 pages. https://doi.org/10.1145/2934328.2934339

[29] Laurynas Šikšnys, Emmanouil Valsomatzis, Katja Hose, and Torben B. Pedersen. 2015. Aggregating and Disaggregating Flexibility Objects. IEEE Transactions on Knowledge and Data Engineering 27, 11 (Nov 2015), 2893-2906. https://doi.org/ 10.1109/TKDE.2015.2445755

[30] Chengke Zhou, Kejun Qian, Malcom Allan, and Wenjun Zhou. 2011. Modeling of the Cost of EV Battery Wear Due to V2G Application in Power Systems. IEEE Transactions on Energy Conversion 26, 4 (Dec 2011), 1041-1050. https: //doi.org/10.1109/TEC.2011.2159977 